\title{
Subsonic flow past localised heating elements in boundary layers
}

DOI:

10.1017/jfm.2017.277

\section{Document Version}

Accepted author manuscript

Link to publication record in Manchester Research Explorer

\section{Citation for published version (APA):}

Aljohani, A., \& Gajjar, J. (2017). Subsonic flow past localised heating elements in boundary layers. Journal of Fluid Mechanics, 821. https://doi.org/10.1017/jfm.2017.277

\section{Published in:}

Journal of Fluid Mechanics

\section{Citing this paper}

Please note that where the full-text provided on Manchester Research Explorer is the Author Accepted Manuscript or Proof version this may differ from the final Published version. If citing, it is advised that you check and use the publisher's definitive version.

\section{General rights}

Copyright and moral rights for the publications made accessible in the Research Explorer are retained by the authors and/or other copyright owners and it is a condition of accessing publications that users recognise and abide by the legal requirements associated with these rights.

\section{Takedown policy}

If you believe that this document breaches copyright please refer to the University of Manchester's Takedown Procedures [http://man.ac.uk/04Y6Bo] or contact uml.scholarlycommunications@manchester.ac.uk providing relevant details, so we can investigate your claim.

\section{OPEN ACCESS}




\title{
Subsonic flow past localised heating elements in boundary layers
}

\author{
A. F. Aljohani ${ }^{1,2}$ and J. S. B. Gajjar ${ }^{2} \dagger$ \\ ${ }^{1}$ Department of Mathematics, Faculty of Science, University of Tabuk, Saudi Arabia \\ ${ }^{2}$ School of Mathematics, University of Manchester, Manchester, M13 9PL,UK
}

(Received $\mathrm{xx}$; revised $\mathrm{xx}$; accepted $\mathrm{xx}$ )

The problem of subsonic flow past micro-electro-mechanical-type (MEMS-type) heating elements placed on a flat surface, where the MEMS devices have hump-shaped surfaces, is investigated using triple deck theory. The compressible Navier-Stokes equations supplemented by the energy equation are considered in the limit that the Reynolds number is large. The triple deck problem is formulated and the linear and non-linear analysis and results are presented. The current work is a generalisation of the problem discussed by (Koroteev \& Lipatov 2012, 2013) where the MEMS devices have flat shaped surfaces. The results show the hump-shaped heating elements enhance large drops in pressure and peaks and troughs in the skin-friction over the centre of the hump as compared to the flat shaped devices which may be useful for controlling the flow.

Key words: Authors should not enter keywords on the manuscript, as these must be chosen by the author during the online submission process and will then be added during the typesetting process (see http://journals.cambridge.org/data/relatedlink/jfmkeywords.pdf for the full list)

\section{Introduction}

Flow separation from the surface of a rigid body is one of the most interesting phenomena in fluid motion. This separation causes major effects in flow fields. Investigating the separation and its consequences is of particular importance in understanding flows at high Reynolds numbers. The explanation of the phenomena of separation lies in the theory of the boundary layer, in particular triple deck theory, see Stewartson \& Williams (1969), Neiland (1969), Messiter (1970). In recent years, there has been an increasing interest in using micro-electro-mechanical-system (MEMS) devices to control the separation of flow at high Reynolds numbers. Based on triple deck theory, we investigate subsonic flow past MEMS type heating elements placed on a flat surface in which the heating element has a hump shape. The current work reduces to the problem investigated by for example Koroteev \& Lipatov (2012), Koroteev \& Lipatov (2013) when the MEMS devices have zero hump height. Apart from MEMS devices, another motivation for the current work is to try and understand how local hot spots or cold bumps affect separation.

$\dagger$ Email address for correspondence: jitesh.gajjar@manchester.ac.uk 


\section{Problem formulation}

Consider a subsonic viscous flow of a perfect gas past a semi-infinite flat plate on which there is a small heating element. In cross-section the hump has dimensions that are small compared with those of the oncoming boundary layer along the plate. The Reynolds number $R e=\rho_{\infty} U_{\infty} L / \mu_{\infty}$ is asymptotically large, where $\rho_{\infty}, U_{\infty}$ and $\mu_{\infty}$ are the density, the stream-wise velocity, and the dynamic viscosity coefficient respectively in the undisturbed flow above the surface where the heated section is located, and $L$ is the distance from the leading edge to the energy release domain. Let $R e^{-\frac{1}{2}}=\varepsilon$. We assume that the hump has length of $O\left(L \varepsilon^{\frac{3}{4}}\right)$ and height of $O\left(L \varepsilon^{\frac{5}{4}}\right)$. In particular, we consider humps that have profiles $\frac{y^{*}}{L \varepsilon^{\frac{5}{4}}}=\bar{h} F\left(\frac{x^{*}}{L \varepsilon^{\frac{3}{4}}}\right)$ where $\bar{h}$ is $O(1)$ and the function $F$ is such that $G(X)=\bar{h} F(X)$ is of order 1. Variables of the governing Navier-Stokes and energy equations are non-dimensionalised with $U_{\infty}, L$ and $\rho_{\infty}$ being the characteristic velocity, length and density scales, respectively. Then, we use the following dimensionless variables

$$
\begin{gathered}
(x, y)=\frac{1}{L}\left(x^{*}, y^{*}\right), \quad(u, v)=\frac{1}{U_{\infty}}\left(u^{*}, v^{*}\right), \quad T=\frac{T^{*} R}{U_{\infty}^{2}}, \\
p=\frac{p^{*}-p_{\infty}}{\rho_{\infty} U_{\infty}^{2}}, \quad \mu=\frac{\mu^{*}}{\mu_{\infty}}, \quad \text { and } \quad \rho=\frac{\rho^{*}}{\rho_{\infty}} .
\end{gathered}
$$

Here $(x, y)$ are the Cartesian coordinates, $(u, v)$ the corresponding velocity components, $p$ is the pressure, $T$ the temperature, $\mu$ the viscosity, $\rho$ the density and $R$ is the gas constant and $p_{\infty}$ is the free-stream pressure. We also introduce the free-stream Mach $M_{\infty}=U_{\infty} / c_{\infty}$ where $c_{\infty}=\sqrt{\gamma p_{\infty} / \rho_{\infty}}$.

Neiland (1971) has discussed the arguments leading to the triple-deck scales and Lipatov (2006), Koroteev \& Lipatov (2012), Koroteev \& Lipatov (2013) in particular have explained how small changes in the surface temperature lead to a nonlinear interaction within the triple-deck. The interested reader is referred to these and other papers for more details of the governing equations and expansions. Using this as our starting point, the method of matched asymptotic expansions is used to obtain equations of the interaction problem. In our case the main difference between the current and previous work is that we also require no slip on the hump surface. In particular in the lower deck the independent variables and expansions are given by:

$$
\begin{gathered}
x=1+\varepsilon^{\frac{3}{4}} X, \quad y=\varepsilon^{\frac{5}{4}} y_{3}, \\
u=\varepsilon^{\frac{1}{4}} u_{3}+\ldots, \quad v=\varepsilon^{\frac{3}{4}} v_{3}+\ldots, \quad p=\varepsilon^{\frac{1}{2}} p_{3}+\ldots, \\
\rho=\rho_{3}+\ldots, \quad \text { and } \quad T=T_{3}+\ldots .
\end{gathered}
$$

Substitution into the Navier-Stokes equations leads to the lower-deck problem

$$
\begin{gathered}
u_{3} \frac{\partial \rho_{3}}{\partial X}+v_{3} \frac{\partial \rho_{3}}{\partial y_{3}}+\rho_{3} \frac{\partial u_{3}}{\partial X}+\rho_{3} \frac{\partial v_{3}}{\partial y_{3}}=0 \\
\rho_{3}\left(u_{3} \frac{\partial u_{3}}{\partial X}+v_{3} \frac{\partial u_{3}}{\partial y_{3}}\right)=-\frac{\partial p_{3}}{\partial X}+\frac{\partial}{\partial y_{3}}\left(\mu \frac{\partial u_{3}}{\partial y_{3}}\right) \\
\frac{\partial p_{3}}{\partial y_{3}}=0, \quad \rho_{3} T_{3}=1 \\
\rho_{3}\left(u_{3} \frac{\partial T_{3}}{\partial X}+v_{3} \frac{\partial T_{3}}{\partial y_{3}}\right)=\frac{\partial}{\partial y_{3}}\left(\frac{\mu}{P r} \frac{\partial T_{3}}{\partial y_{3}}\right)
\end{gathered}
$$


with the boundary and matching conditions

$$
\begin{gathered}
u_{3} \rightarrow \lambda\left(y_{3}+A(X)\right), \quad T_{3} \rightarrow T_{B}(x, 0) \quad \text { as } \quad y_{3} \rightarrow \infty, \\
u_{3}=v_{3}=0, \quad T_{3}=T_{w}(X) \quad \text { on } \quad y_{3}=G(X) .
\end{gathered}
$$

We also have the interaction law

$$
p_{3}(X)=\frac{1}{\pi \sqrt{1-M_{\infty}^{2}}} \int_{-\infty}^{\infty} \frac{A^{\prime}(\zeta)}{X-\zeta} d \zeta .
$$

Here $A(X)$ is the displacement function, $\lambda=\frac{\partial U_{B}}{\partial y_{3}}\left(1, y_{3}=0\right)$ is the basic shear of the oncoming boundary layer profile $U_{B}(x, y)$, and $T_{B}(x, y)$, is the basic temperature profile, $G(X)$ is the physical hump shape. As a result after further employing the HowarthDorodnitsyn transformation followed by the Prandtl transformation, using the Chapman viscosity law, setting the Prandtl number to be unity, and scaling to remove some constants, the governing equations and boundary conditions for subsonic flow in the lower deck can be shown to be given by:

$$
\begin{gathered}
\frac{\partial u_{b}}{\partial x_{b}}+\frac{\partial v_{b}}{\partial y_{b}}=0 \\
u_{b} \frac{\partial u_{b}}{\partial x_{b}}+v_{b} \frac{\partial u_{b}}{\partial y_{b}}+T_{b} \frac{\partial p_{b}}{\partial x_{b}}=\frac{\partial^{2} u_{b}}{\partial y_{b}^{2}} \\
u_{b} \frac{\partial T_{b}}{\partial x_{b}}+v_{b} \frac{\partial T_{b}}{\partial y_{b}}=\frac{\partial^{2} T_{b}}{\partial y_{b}^{2}}, \\
p_{b}\left(x_{b}\right)=\frac{1}{\pi} f_{-\infty}^{\infty} \frac{\mathcal{K}^{\prime}(s)}{x_{b}-s} d s
\end{gathered}
$$

with the no-slip conditions on $y_{b}=0$ given by

$$
u_{b}\left(x_{b}, 0\right)=v_{b}\left(x_{b}, 0\right)=0,
$$

and the prescribed wall temperature

$$
T_{b}\left(x_{b}, 0\right)=T_{w}\left(x_{b}\right)
$$

The boundary conditions far from the surface $\left(y_{b} \rightarrow \infty\right)$ are given by

$$
u_{b} \rightarrow y_{b}+\mathcal{A}\left(x_{b}\right)+H\left(x_{b}\right), \quad \mathcal{K}\left(x_{b}\right)=\mathcal{A}\left(x_{b}\right)+\int_{0}^{\infty}\left(1-T_{b}\left(x_{b}, \eta\right)\right) d \eta, \quad T_{b}\left(x_{b}, y_{b}\right) \rightarrow 1
$$

and far upstream

$$
\mathcal{A}(-\infty)=0
$$

The interaction condition (2.8) can be represented in another form as mentioned in Smith (1973), as

$$
\mathcal{K}^{\prime \prime}\left(x_{b}\right)=-\frac{1}{\pi} \int_{-\infty}^{\infty} \frac{p_{b}^{\prime}(s)}{x_{b}-s} d s .
$$

Here $x_{b}$ is the scaled streamwise coordinate in the triple-deck, $y_{b}$ the scaled lowerdeck coordinate (following the use of the Dorodnitsyn-Howarth transformation), $u_{b}\left(x_{b}, y_{b}\right), v_{b}\left(x_{b}, y_{b}\right)$ are the scaled lower-deck velocity components, $T_{b}\left(x_{b}, y_{b}\right)$ is the scaled temperature, $p_{b}\left(x_{b}\right)$ is the induced pressure, $\mathcal{K}\left(x_{b}\right)$ is the total displacement of stream lines and $\mathcal{A}\left(x_{b}\right)$ the displacement of stream lines caused by the viscosity of the fluid, $T_{w}\left(x_{b}\right)$ represents the prescribed wall temperature. The function $H\left(x_{b}\right)$ is the 
shape of the element surface which is related to the physical hump shape $G\left(x_{b}\right)$ in the original variables by

$$
H\left(x_{b}\right)=\int_{0}^{G\left(x_{b}\right)} \rho_{3}\left(x_{b}, y_{b}\right) d y_{b} .
$$

The main difference between the present work and that of Koroteev \& Lipatov (2012), Koroteev \& Lipatov (2013) is that $H\left(x_{b}\right)=0$ in the latter.

\section{Linear theory}

In general to solve the equations (2.5)-(2.13) for variations of the temperature $\triangle T$ of $O(1)$, and $H\left(x_{b}\right)$ of $O(1)$ requires a numerical method. For small variations of the temperature and hump height we may study the problem analytically in the linear approximation. Consider small variations of the temperature i.e. $\sigma=\Delta T \ll 1$. We may linearise the equations (2.5)-(2.13) related to the undisturbed boundary layer profile by expanding the flow variables as follows:

$$
\begin{gathered}
u_{b}=y_{b}+\sigma \tilde{U}+O\left(\sigma^{2}\right), \quad v_{b}=\sigma \tilde{V}+O\left(\sigma^{2}\right), \\
\mathcal{K}=\sigma \mathcal{K}_{1}+O\left(\sigma^{2}\right), \quad T_{b}=1+\sigma \tilde{T}+O\left(\sigma^{2}\right), \\
p_{b}=\sigma \tilde{P}+O\left(\sigma^{2}\right), \quad H=\sigma \tilde{H}+O\left(\sigma^{2}\right), \quad \text { and } \quad \mathcal{A}=\sigma \mathcal{A}_{1}+O\left(\sigma^{2}\right)
\end{gathered}
$$

as $\sigma \rightarrow 0$. After substituting the expansions, (3.1)-(3.3), into equations and boundary conditions (2.5)-(2.13) we obtain the following linearised problem

$$
\begin{gathered}
\frac{\partial \tilde{U}}{\partial x_{b}}+\frac{\partial \tilde{V}}{\partial y_{b}}=0, \\
\frac{\partial \tilde{U}}{\partial x_{b}}+\tilde{V}+\frac{\partial \tilde{P}}{\partial x_{b}}=\frac{\partial^{2} \tilde{U}}{\partial y_{b}^{2}}, \\
y_{b} \frac{\partial \tilde{T}}{\partial x_{b}}=\frac{\partial^{2} \tilde{T}}{\partial y_{b}^{2}} .
\end{gathered}
$$

The linearised boundary conditions are given by

$$
\begin{gathered}
\tilde{U}\left(x_{b}, 0\right)=0, \quad \tilde{V}\left(x_{b}, 0\right)=0, \quad \tilde{T}\left(x_{b}, 0\right)=\tilde{T}_{w}\left(x_{b}\right) \\
\tilde{U} \rightarrow \mathcal{A}_{1}\left(x_{b}\right)+\tilde{H}\left(x_{b}\right), \quad \mathcal{K}_{1}\left(x_{b}\right)=-\int_{0}^{\infty} \tilde{T}(x, \eta) d \eta+\mathcal{A}_{1}\left(x_{b}\right), \\
\tilde{T}\left(x_{b}, \infty\right) \rightarrow 0 \quad \text { as } \quad y_{b} \rightarrow \infty, \\
\mathcal{A}_{1}(-\infty)=0 \quad \text { and } \quad \mathcal{K}_{1}^{\prime \prime}\left(x_{b}\right)=-\frac{1}{\pi} f_{-\infty}^{\infty} \frac{\tilde{P}^{\prime}(s)}{x_{b}-s} d s .
\end{gathered}
$$

We will use Fourier Transforms to solve the above equations. The Fourier Transform of $g\left(x_{b}\right)$ and the inverse transform are defined by

$$
\hat{g}(\omega)=\int_{-\infty}^{\infty} g\left(x_{b}\right) e^{-i \omega x_{b}} d x_{b}, \quad g\left(x_{b}\right)=\frac{1}{2 \pi} \int_{-\infty}^{\infty} \hat{g}(\omega) e^{i \omega x_{b}} d \omega .
$$

After applying the Fourier transformation the linear equations may be solved to obtain 


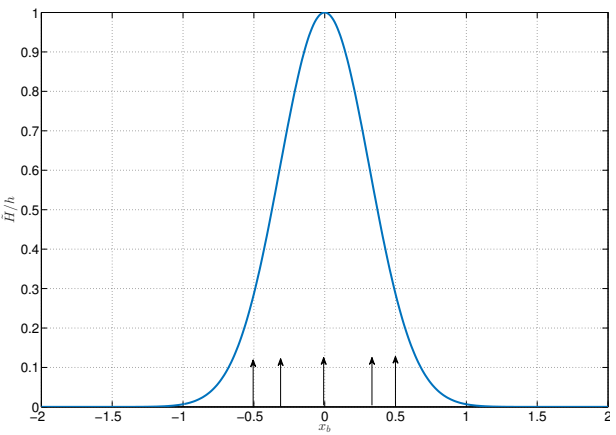

(a)

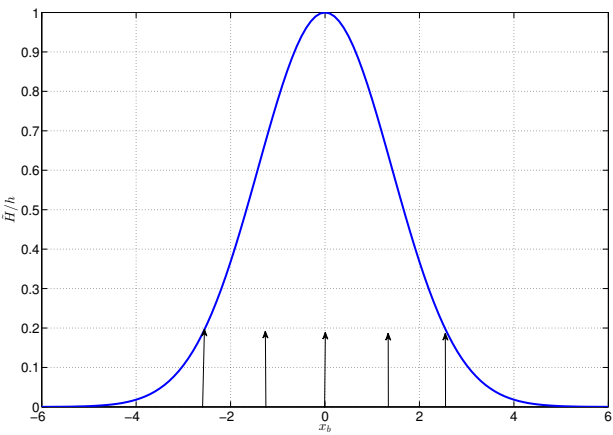

(b)

Figure 1: Plot of the hump shape a) $\tilde{H}\left(x_{b}\right) / \tilde{h}=e^{-5 x_{b}^{2}}$, and b) $\tilde{H}\left(x_{b}\right) / \tilde{h}=e^{-0.25 x_{b}^{2}}$. The arrows depict the extent of the heated region for $\left|x_{b}\right|<a / 2$.

expressions for the perturbed pressure gradient and wall shear distributions as

$$
\frac{\partial \tilde{P}}{\partial x_{b}}=\frac{\operatorname{Ai}^{\prime}(0)}{2 \pi \operatorname{Ai}(0)} \int_{-\infty}^{\infty} \frac{(i \omega)^{\frac{5}{3}} \hat{f}(\omega)+(i \omega)^{2} \hat{H}(\omega) \Lambda}{i \operatorname{sgn}(\omega) \Theta+(i \omega)^{\frac{4}{3}}} e^{i \omega x_{b}} d \omega
$$

and

$$
\frac{\partial \tilde{U}}{\partial y_{b}}\left(x_{b}, 0\right)=\frac{1}{2 \pi} \int_{-\infty}^{\infty} \frac{(i \omega)^{\frac{4}{3}} \hat{f}(\omega)+(i \omega)^{\frac{5}{3}} \hat{H}(\omega) \Lambda}{i \operatorname{sgn}(\omega) \Theta+(i \omega)^{\frac{4}{3}}} e^{i \omega x_{b}} d \omega
$$

where $\Theta=-\frac{\mathrm{Ai}^{\prime}(0)}{\int_{0}^{\infty} \mathrm{Ai}(z) d z}$ and $\Lambda=\frac{\mathrm{Ai}(0)}{\int_{0}^{\infty} \mathrm{Ai}(z) d z}$, and $\hat{f}$ is the transform of the wall temperature $\tilde{T}_{w}\left(x_{b}\right)$. The multi-valued functions are defined such that we have taken a branch cut along the positive imaginary axis for $\omega$.

The expressions for the pressure and wall shear can be inverted for various wall shapes and heating profiles, (see the supplementary material for this paper). For example, if we assume that the heating region is located in $\left|x_{b}\right|<0.5$ and that the wall temperature function is given by

$$
\tilde{T}\left(x_{b}, 0\right)= \begin{cases}0.2 & \left|x_{b}\right|<0.5 \\ 0 & \left|x_{b}\right|>0.5\end{cases}
$$

and that the hump shape is given by

$$
\tilde{H}\left(x_{b}\right)=\tilde{h} \exp \left(-5 x_{b}^{2}\right)
$$

then

$$
\hat{f}(\omega)=\frac{0.4}{\omega} \sin \left(\frac{\omega}{2}\right), \quad \mathcal{F}\left\{\tilde{H}\left(x_{b}\right)\right\}=\hat{H}(\omega)=\tilde{h} \frac{\sqrt{5 \pi}}{5} \exp \left(-\frac{\omega^{2}}{20}\right) .
$$

A plot of the hump profiles used in the linear and nonlinear computations in comparison to the extent of the heated region can be seen in figure 1 . In figures $2 \mathrm{a}$ and $2 \mathrm{~b}$ we present the pressure $\tilde{P}\left(x_{b}\right)$ and the wall shear $\tilde{\tau}=\frac{\partial \tilde{U}}{\partial y_{b}}\left(x_{b}, 0\right)$ distributions for different values of the hump height $\tilde{h}$. In figure 2 a we can see that the pressure increases as $x_{b}$ increases ahead of the hump but drops significantly on reaching the beginning of the heating region at $x_{b}=-0.5$, followed by recovery at the end of the heated region. The wall-shear distribution in figure $2 \mathrm{~b}$ decreases initially but there is a sharp rise and drop 


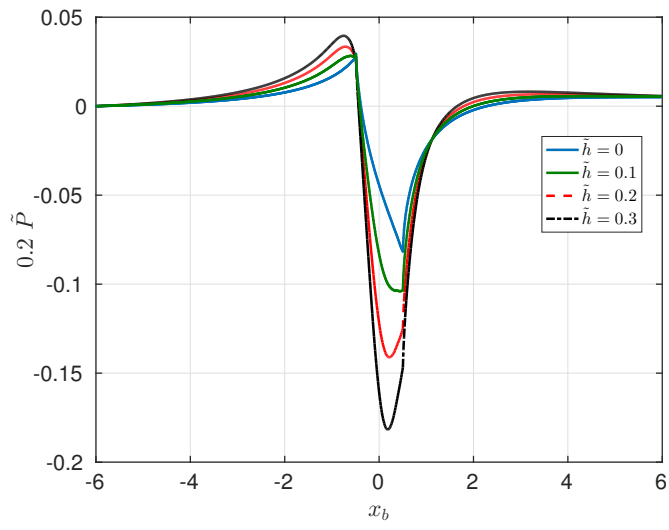

(a)

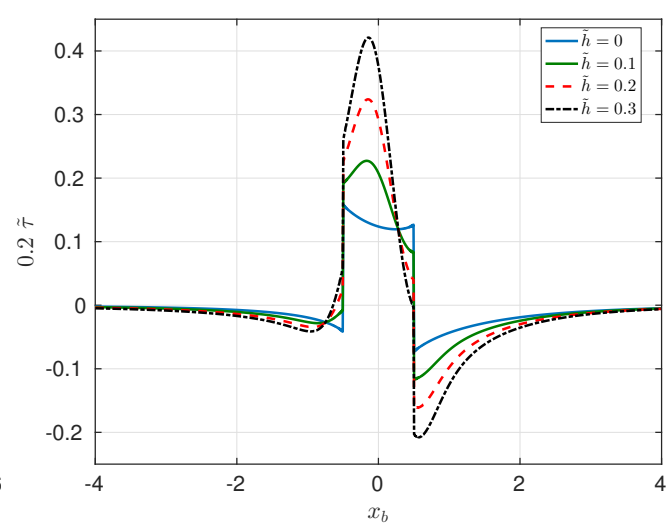

(b)

Figure 2: Distributions of (a) the pressure and (b) wall shear for various values of $\tilde{h}$ in the linear approximation.

on entering and leaving the heated region. The discontinuous changes for $\tilde{h}=0$ at the start and end of the heated region are also seen in the results of Koroteev \& Lipatov (2012). The main difference between the flat plate case with $\tilde{h}=0$ and the hump shape is that the presence of the hump creates a larger pressure drop and much larger rise in the wall shear stress across the heated part as compared to the flat plate case. Also the sharp discontinuous change seen in the pressure and the wall shear distribution for the flat plate case is smoothed out for the hump shaped profile.

\section{The non-linear theory}

\subsection{Numerical method to solve the non-linear problem}

The triple deck equations are non-linear and in order to solve them we can use various numerical methods. In Sychev et al. (1998), different approaches are described to solve these kind of problems. Our method consists of using finite differences in the $x$-direction (stream-wise direction) and Chebyshev collocation method in the $y$-direction (wall normal direction) using the technique as described in Korolev et al. (2002), Logue (2008). Hereafter, the subscript $b$ in the variables is omitted. In the $y$-direction we work with a finite domain $\left[0, y_{\max }\right]$ where $y_{\max }$ is a suitably chosen large value. In the $x$-direction we have a truncated domain $\left[x_{\min }, x_{\max }\right]$. The $y$-component of the node $\left(x_{i}, y_{k}\right)$ is given by the Chebyshev collocation points which are represented using the mapping $y \rightarrow z \subseteq(-1,1)$ in Chebyshev space so that the collocation points are given by $z=z_{j}=-\cos \left(\frac{j \pi}{N}\right), j=0,1, \ldots, N$ and $y=y\left(z_{k}\right)=y_{k}=\frac{1}{2} y_{\max }\left(z_{k}+1\right), k=0,1, \ldots, N$ where $N+1$ is the number of points in the $y$-direction. Finite differences are used to discretise the $x$-direction. The finite difference points are given by $x=x_{i}=x_{\text {min }}+(i-$ 1) $\triangle x, i=1,2, \ldots, M$, where $\triangle x=\frac{x_{\max }-x_{\min }}{M-1}$.

The interaction law equation can be written as, $\frac{\partial p}{\partial x}=-\frac{1}{\pi} \int_{-\infty}^{\infty} \frac{\mathcal{K}^{\prime \prime}(s)}{s-x} d s$. In order to discretize this we use the method as presented in Kravtsova et al. (2005) to isolate the singular part near $s=x$ and utilize local expansions in the vicinity of the singular point. Finally using Newton linearization, and by defining the unknowns variables as

$$
\underline{\mathcal{L}}_{i}=\left(\underline{U}_{i}, \underline{T}_{i}, P_{i}, \mathrm{~A}_{i}, \mathrm{~K}_{i}\right)^{T}
$$


the linearized discrete equation system can be written as

$$
A_{i} \underline{\mathcal{L}}_{i-2}+B_{i} \underline{\mathcal{L}}_{i-1}+C_{i} \underline{\mathcal{L}}_{i}+F_{i} \underline{\mathcal{L}}_{i+1}+E_{i} \underline{\mathcal{L}}_{i+2}=\underline{r h s}_{i} .
$$

Here the block matrices $A_{i}, B_{i}, C_{i}, F_{i}$ and $E_{i}$ are of size $(2 N+5) \times(2 N+5)$. Note that from the interaction law the additional terms which do not contribute to the block pentadiagonal structure above are transferred to the right hand side treating them as known quantities. This discrete system is applied to $3 \leqslant i \leqslant(M-2)$, while for $i<3$ we impose values that are given by undisturbed boundary layer profiles, i.e $U=y, T=1, K=0$ and $P=0$. For $i>(M-2)$ one sided differences are used. A detailed description of the full discretised equations system can be found in Aljohani (2016). The linear equations are solved directly using a solver which exploits the block pentadiagonal sparsity pattern of the matrices in (4.1).

\subsection{Results and discussion}

In this section we present results from the numerical solution of the nonlinear triple deck problem for subsonic flow past a MEMS-type heating element. The results depend on the hump shape which is chosen as

$$
H\left(x_{b}\right)=h e^{-0.25 x_{b}^{2}}
$$

with parameter $h$ and also on the wall temperature given by

$$
T_{w}\left(x_{b}\right)=\left\{\begin{array}{ll}
1+\triangle T & \left|x_{b}\right| \leqslant \frac{a}{2} \\
1 & \left|x_{b}\right|>\frac{a}{2}
\end{array},\right.
$$

with the parameters $\triangle T$ being the amplitude of perturbations of the temperature, and $a$ is the extent of the heated region. Note that for the nonlinear results the hump shape is vanishingly small for $\left|x_{b}\right|>5$ as can be seen in figure 1 .

Koroteev \& Lipatov (2012) investigated the problem of subsonic laminar boundary layer where local heated elements are placed along the surface. A comparison of the wall shear distribution obtained in Koroteev \& Lipatov (2012) and by the present numerical method is shown in figure 3 with $\triangle T=0.2, h=0$ and $a=14$. Excellent agreement between the results can be seen in figure 3. The previous work results, here and later, are reproduced using a digitizing software developed by Rohatgi (2010). Various grid size and other checks are carried out to justify that the results are consistent as detailed in Aljohani (2016).

In the remainder of the results presented below, we have fixed $a=10$. A comparison of the linear and nonlinear results is shown in figures $4 \mathrm{a}$ and $4 \mathrm{~b}$ where we present the normalised pressure and wall shear distributions with $\triangle T=0.3$ and $h=0.2$. It can be seen that for these values of $h$ the linear approximation is not that different from the nonlinear results. One additional feature which can be noticed in figure $4 \mathrm{a}$ as compared to figure $2 \mathrm{a}$ is the rise and dip in pressure on the rear portion of the hump. In figure $2 \mathrm{a}$ the heating was confined to a small part of the hump whereas in figure 4a the heating extends to $x_{b}=5$ which effectively coincides with the start of the flat plate region. Once the heating is switched off the pressure rises rapidly in both cases. In figure $4 \mathrm{~b}$ the rise and fall in the pressure over the rear portion of the hump also gives rise to a dip and rise in the wall shear in the same region. In figure 5 we have plotted the maximum and minimum values of the wall shear for a larger value of $h=0.6$, for varying values of $\Delta T$. Significant differences between the linear and nonlinear results only show up after $\Delta T$ exceeds 1 for the maximum wall shear.

Further nonlinear results are presented in figures $6 \mathrm{a}$ and $6 \mathrm{~b}$, keeping the hump size fixed 


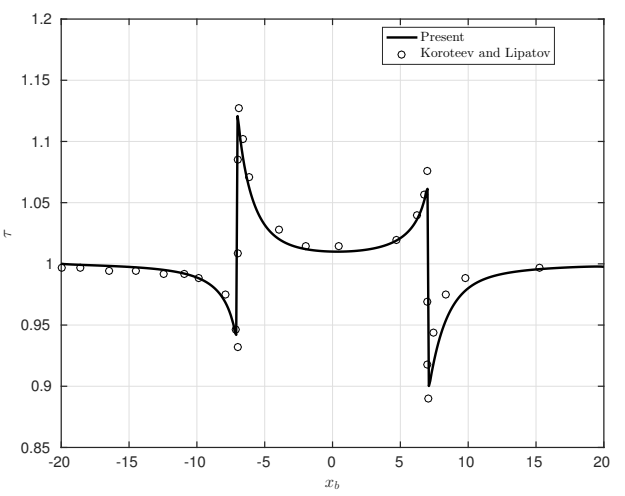

Figure 3: A comparison of the present results with Koroteev \& Lipatov (2012) for the wall shear with $\triangle T=0.2, a=14$ and $h=0$.

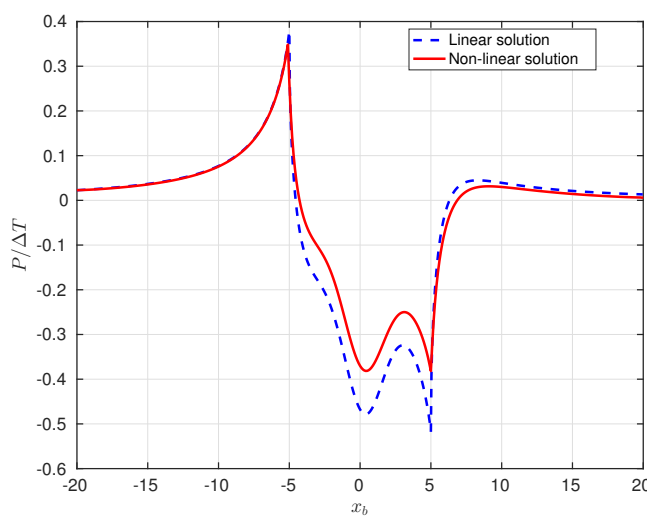

(a)

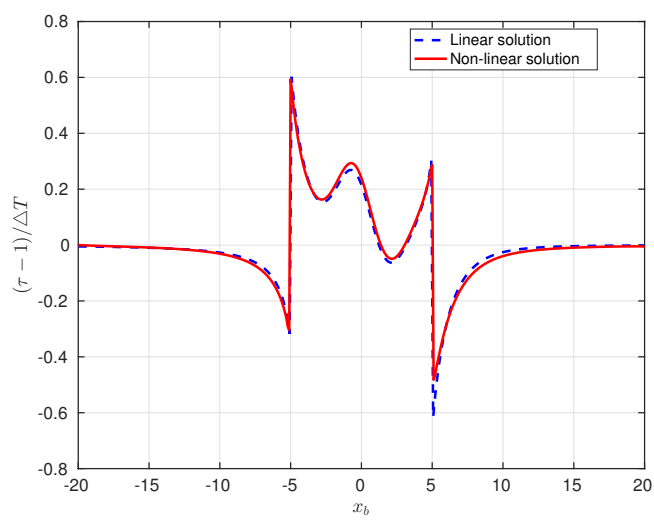

(b)

Figure 4: Comparisons of the non-linear and linear results for (a) Pressure and (b) Wall shear with $\triangle T=0.3, a=10$ and $h=0.2$

with $h=0.5$ and with the wall temperature varying. It can be observed that increasing $\triangle T$ increases the pressure and wall shear maximum values which are located on the upstream edge of the heated region and decreases the pressure and wall shear minimum values which are located on the downstream edge of the heating region. Once the heating starts, the flow becomes more strongly attached over the hump, although there is still a local rise and fall in the pressure over the rear protion of the hump. Figures $7 \mathrm{a}$ and $7 \mathrm{~b}$ show distributions of the pressure and skin friction presented keeping the heating the same with $\triangle T=0.4$ while the hump height $h$ varies. It can be noticed that increasing $h$ leads to shifting the maximum and minimum values of the pressure and wall shear to be in the neighbourhood of the centre of the element rather being on its edges. Increasing the hump height also promotes a much stronger adverse pressure gradient on the rear portion of the hump. 


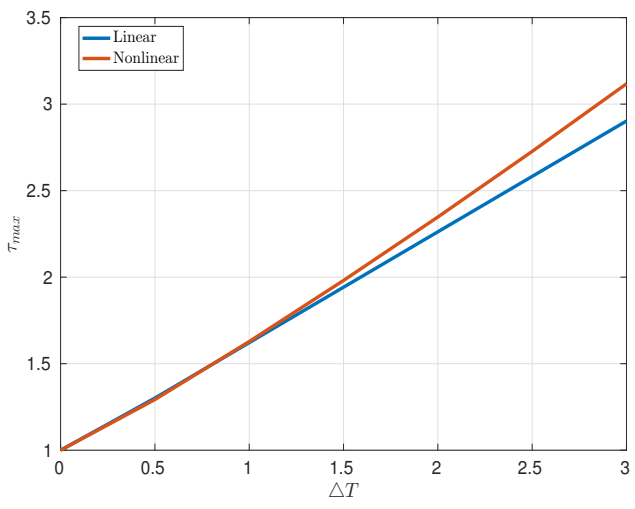

(a)

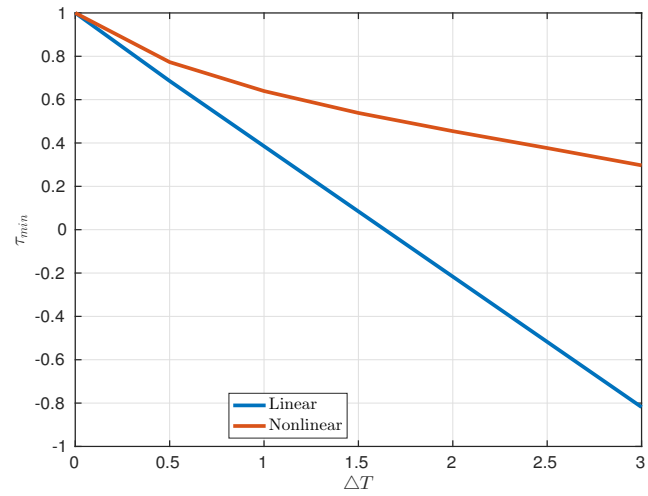

(b)

Figure 5: Amplitudes of perturbations of the temperature $\triangle T$ versus (a) maximum wall shear values and (b) minimum wall shear values, with $a=10$ and $h=0.6$.

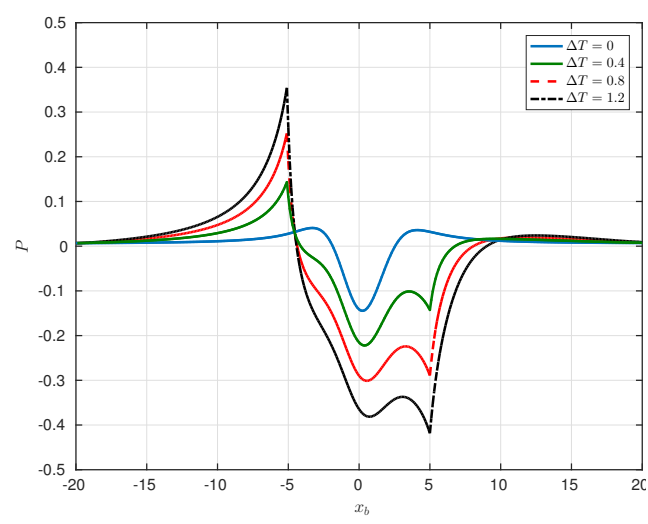

(a)

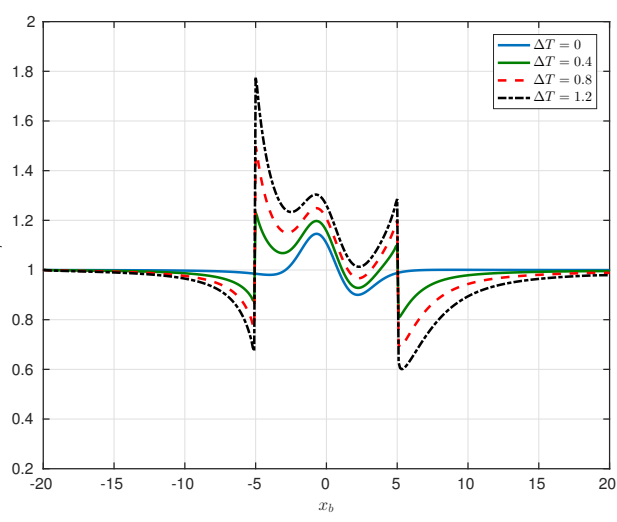

(b)

Figure 6: (a) Pressure and (b) Wall shear for $h=0.5$ and various values of $\triangle T$.

\section{Conclusion}

The linear and non-linear problems of subsonic flow past a heating element have been discussed and numerical methods were used to solve the linear and non-linear problems. The results for the heated hump are new and have not been seen before. Our results have been shown to compare well with previous work for the flat plate case. We have shown that having a shorter hump with heating applied only to the middle part of the hump smooths out the sharp variations in the wall shear and temperature near the start and end of the heated region. Further a shorter taller hump generally enhances the favourable properties of the heated element in creating a stronger attached flow. Secondly for longer humps, the presence of the hump creates large peaks and troughs in the pressure and wall shear particularly near the centre of the hump. These are not observed in the flat plate case.

Clearly the size and shape of the hump and the location of the heating region are 


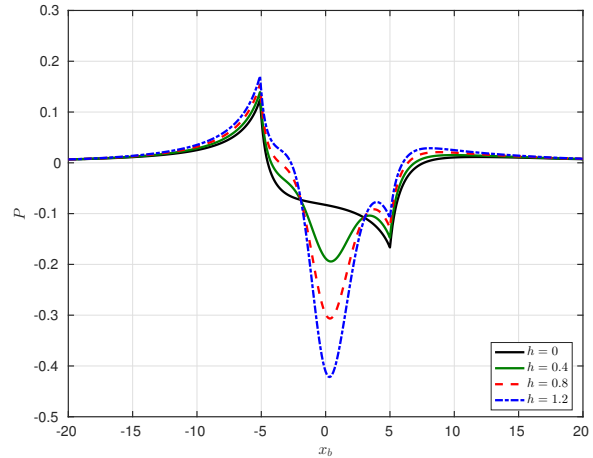

(a)

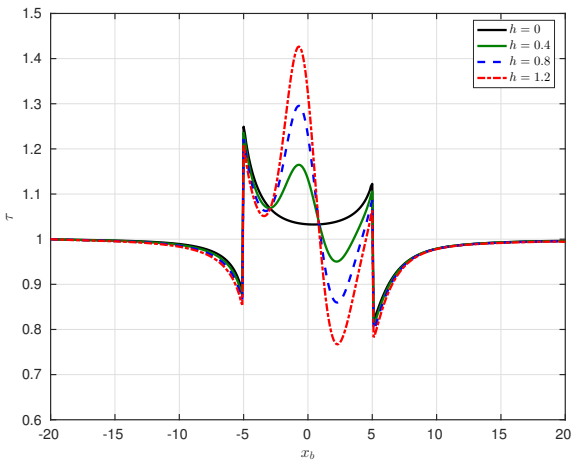

(b)

Figure 7: (a) Pressure and (b) Wall Shear for $\triangle T=0.4$ and various values of $h$.

important factors to be taken into consideration in designing suitable MEMS devices which can be used to control the flow and separation.

\section{REFERENCES}

Aljohani, A. F. 2016 Applications of triple deck theory to study the flow over localised heating elements in boundary layers. PhD thesis, University of Manchester.

Korolev, G.L., GajJar, J.S.B. \& Ruban, A.I. 2002 Once again on the supersonic flow separation near a corner. J. Fluid Mech. .

Koroteev, M. V. \& Lipatov, I. I. 2012 Local temperature perturbations of the boundary layer in the regime of free viscousinviscid interaction. J. Fluid Mech. 707, 595-605.

Koroteev, M. V. \& Lipatov, I. I. 2013 Steady subsonic boundary layer in domains of local surface heating. App. Math. Mech. 77, 486-493.

Kravtsova, M. A., Zametaev, V. B. \& Ruban, A. I. 2005 An effective numerical method for solving viscous-inviscid interaction problems. Phil. Trans. R. Soc. Lond. 363, 1157-1167.

Lipatov, I. I. 2006 Disturbed boundary layer flow with local time-dependent surface heating. Fluid Dyn. 41, 55-65.

Logue, R.P. 2008 Stability and bifurcations governed by the triple-deck and related equations. PhD thesis, University of Manchester.

Messiter, A. F. 1970 Boundary-layer flow near the trailing edge of a flat plate. SIAM J. Appl. Maths 18, 241-257.

NeILAnd, V. Y. 1969 Theory of laminar boundary layer separation in supersonic flow. Izv. Akad. Nauk SSSR, Mech. Zhidk. Gaza 4, 53-57.

NeILAND, V. Y. 1971 The asymptotic theory of the interaction of a supersonic flow with a boundary layer. Izv. AN SSSR, Mekh. Zhidk. i Gaza 4, 41-47.

Rohatgi, A. 2010 Webplotdigitizer. http: //arohatgi. info/WebPlotDigitizer.

Smith, F. T. 1973 Laminar flow past a small hump on a plate. J. Fluid Mech. 57, 803-824.

Stewartson, K. \& Williams, P. G. 1969 Self-induced separation. Proc. R. Soc. Lond. A 312, $181-206$.

Sychev, V. V., Ruban, A. I., Sychev, Vic. V. \& Korolev, G. L. 1998 Asymptotic Theory of Separated Flows. Cambridge University Press. 